\title{
STATIC ELECTROMAGNETIC FIELDS IN MONOTONE MEDIA
}

\author{
RAINER PICARD \\ Department of Mathematical Sciences, University of Wisconsin \\ Milwaukee, Wisconsin 53201, U.S.A.
}

\begin{abstract}
The paper considers the static Maxwell system for a Lipschitz domain with perfectly conducting boundary. Electric and magnetic permeability $\varepsilon$ and $\mu$ are allowed to be monotone and Lipschitz continuous functions of the electromagnetic field. The existence theory is developed in the framework of the theory of monotone operators.
\end{abstract}

0. Introduction. In the static case Maxwell's equations of the electromagnetic field, described by the electric field $E$ and the electric induction (displacement) $D$, and by the magnetic field $H$ and the magnetic induction $B$, are

$$
\operatorname{curl} E=j, \quad \operatorname{curl} H=0, \quad \operatorname{div} D=q, \quad \operatorname{div} B=0,
$$

where $j$ is a given current density and $q$ is a known charge density. The connection between electric and magnetic field on the one hand and electric and magnetic induction on the other hand is usually given in terms of the so-called material relations:

$$
D=\varepsilon(E, H), \quad B=\mu(E, H) .
$$

In the following we will focus on the system of linear differential equations (0.1) in conjunction with material relations of the type $(0.2)$ in the case that the medium described by these material relations is confined to a bounded domain $\Omega$ with a perfectly conducting boundary $\partial \Omega$. The exterior domain case can be dealt with by similar considerations (see [11] for details). The boundary conditions modelling a perfectly conducting container of the medium in question are

$$
n \times E=0 \text { and } n \cdot B=0 \quad \text { on } \partial \Omega ;
$$

here $n$ denotes the exterior normal to $\partial \Omega$.

In order to develop the solution theory for the boundary value problem (0.1)(0.3), we first have to reformulate the equalities involved in suitable spaces. A high degree of convenience and elegance can be achieved by the use of differential 
forms in formulating the problem and we shall make use of this opportunity. It is a simple task to translate the above boundary value problem for static electromagnetic fields into the language of differential forms. We shall briefly outline this transition for the benefit of those readers who are less comfortable with differential forms. Consider $E, D$ as differential forms of degree one and $H$, $B$ as such forms of degree two, i.e.

$$
\begin{aligned}
& E(x)=E_{1}(x) d x^{1}+E_{2}(x) d x^{2}+E_{3}(x) d x^{3}, \\
& D(x)=D_{1}(x) d x^{1}+D_{2}(x) d x^{2}+D_{3}(x) d x^{3} \\
& H(x)=H_{1}(x) d x^{2} \wedge d x^{3}+H_{2}(x) d x^{3} \wedge d x^{1}+H_{3}(x) d x^{1} \wedge d x^{2}, \\
& B(x)=B_{1}(x) d x^{2} \wedge d x^{3}+B_{2}(x) d x^{3} \wedge d x^{1}+B_{3}(x) d x^{1} \wedge d x^{2} .
\end{aligned}
$$

Here " $\wedge$ " denotes the so-called wedge product which is characterized by its formal alternating property:

$$
d x^{i} \wedge d x^{j}=-d x^{j} \wedge d x^{i},
$$

and the fact that it is associative and linear over the module of functions (i.e. linear over $\mathbb{R}$ for fixed $x \in \mathbb{R}^{3}$ ). Note that in particular (0.4) implies $d x^{i} \wedge d x^{i}=$ $0, i=1,2,3$. The $d x^{i}$ are interpreted as the dual elements to the canonical basis of $\mathbb{R}^{3}$, and alternating products of these as multilinear maps giving the parity of the permutation of the elements of the canonical basis relative to the order in which the indices appear in the wedge product.

The differential operators curl and div (divergence) can now be written in terms of the exterior derivative using the duality between differential forms of degree one and two as expressed by the Hodge star operator $*$.

In the present situation of the three-dimensional Euclidean space the Hodge star operator can be expressed by the properties of being linear over the module of functions and satisfying

$$
\begin{aligned}
* d x^{i} & =d x^{i+1} \wedge d x^{i+2} \quad(i=1,2,3 \bmod 3), \\
* 1 & =d x^{1} \wedge d x^{2} \wedge d x^{3}, \\
* * & =1 \quad \text { (here } 1 \text { denotes the identity). }
\end{aligned}
$$

As an illustration of these properties we note that e.g.

$$
(* H)(x)=H_{1}(x) d x^{1}+H_{2}(x) d x^{2}+H_{3}(x) d x^{3},
$$

and

$$
(* E)(x)=E_{1}(x) d x^{2} \wedge d x^{3}+E_{2}(x) d x^{3} \wedge d x^{1}+E_{3}(x) d x^{1} \wedge d x^{2} .
$$

The formal operation of the exterior derivative $d$ can be recalled most easily by considering $d$ as a formal 1-form:

$$
d=\partial_{1} d x^{1}+\partial_{2} d x^{2}+\partial_{3} d x^{3}
$$

here $\partial_{i}, i=1,2,3$, denotes the partial derivative with respect to the $i$ th variable. 
Then the action of $d$ can be written as the formal alternating product

$$
d \omega=d \wedge \omega
$$

where $\omega$ denotes an arbitrary differential form (with differentiable coefficients).

To illustrate the usefulness of the exterior derivative in the present context let us consider the exterior derivatives of $E$ and $B$. We have (using the summation convention)

$$
\begin{aligned}
d E= & d \wedge E=\left(\partial_{i} d x^{i}\right) \wedge\left(E_{j} d x^{j}\right)=\partial_{i} E_{j} d x^{i} \wedge d x^{j} \\
= & \left(\partial_{1} E_{2}-\partial_{2} E_{1}\right) d x^{1} \wedge d x^{2}+\left(\partial_{2} E_{3}-\partial_{3} E_{2}\right) d x^{2} \wedge d x^{3} \\
& +\left(\partial_{1} E_{3}-\partial_{3} E_{1}\right) d x^{1} \wedge d x^{3} \\
= & \left(\partial_{i+1} E_{i+2}-\partial_{i+2} E_{i+1}\right) d x^{i+1} \wedge d x^{i+2}
\end{aligned}
$$

(summation over indices $\bmod 3$ ), and

$$
\begin{aligned}
d B & =d \wedge B=\left(\partial_{i} d x^{i}\right) \wedge\left(B_{j} d x^{j+1} \wedge d x^{j+2}\right) \\
& =\partial_{i} B_{j} d x^{i} \wedge d x^{j+1} \wedge d x^{j+2} \\
& =\partial_{i} B_{i} d x^{i} \wedge d x^{i+1} \wedge d x^{i+2}=\partial_{i} B_{i} d x^{1} \wedge d x^{2} \wedge d x^{3} .
\end{aligned}
$$

These calculations illustrate that the exterior derivative can be utilized to express $\operatorname{curl} E$ and $\operatorname{div} B$ conveniently in the language of differential forms. The same can be done for transcribing $\operatorname{div} D$ and curl $H$ using the Hodge star operator. Letting $\delta$ denote the operator $(-1)^{q-1} * d *$ (where $q$ indicates the degree of the form this operator is applied to), we have $\delta D$ and $-\delta H$ in place of these vector analytical expressions.

By transcribing $j$ as a 2-form: $j=j_{k} d x^{k+1} \wedge d x^{k+2}$, and $q$ as a 0 -form (i.e. a function), the initial system of differential equations can be written as

$$
d E=j, \quad \delta H=0, \quad \delta D=q, \quad d B=0 .
$$

Since differential forms of different degree can easily be distinguished, we may, as a first benefit of our transcription, write $\omega=E+B$ and $\eta=D+H$ in the sense of a direct sum. With this convention we can simplify (0.8) to just two equations:

$$
d \omega=j \quad \text { and } \quad \delta \eta=q .
$$

The next observation is that the boundary conditions (0.3) take on a very simple form. By using the invariance properties of differential forms with respect to coordinate transformations and assuming for now that the boundary can be described in local coordinates by $x_{3}=0$, the restriction of $\omega$ to the submanifold $x_{3}=0$ is

$$
E_{1}\left(x_{1}, x_{2}, 0\right) d x^{1}+E_{2}\left(x_{1}, x_{2}, 0\right) d x^{2}+B_{3}\left(x_{1}, x_{2}, 0\right) d x^{1} \wedge d x^{2} .
$$

Thus the boundary conditions (0.3), having in these coordinates the form

$$
E_{1}\left(x_{1}, x_{2}, 0\right)=E_{2}\left(x_{1}, x_{2}, 0\right)=B_{3}\left(x_{1}, x_{2}, 0\right)=0,
$$


can simply be expressed by saying that $\omega$ vanishes on the boundary as a submanifold of $\mathbb{R}^{3}$, i.e.

$$
\omega=0 \quad \text { on } \partial \Omega .
$$

The connection between $\omega$ and $\eta$ has to be extracted from the material relations (which we assume to be possible) in the form

$$
\eta=\varepsilon(\omega),
$$

where for simplicity we have re-used the symbol $\varepsilon$ with little danger of confusion.

In order to develop the discussion of problem (0.9)-(0.11) we will have to introduce suitable (real) Hilbert spaces to give a rigorous interpretation to our boundary value problem. Our base space will be an $L_{2}$-type space with inner product composed of the parts

$$
(\alpha, \beta)_{q}=\int_{\Omega} \alpha \wedge * \beta, \quad \alpha, \beta q \text {-forms, } q=0,1,2,3 .
$$

Note that $\alpha \wedge * \beta$ is always a 3 -form and the integral can be understood as an ordinary volume integral by using $d x^{1} d x^{2} d x^{3}$ as (Cartesian) volume element. This underlying space $L_{2}(\Omega)$ is defined as the completion of (direct) sums of differential forms of all degrees $q=0,1,2,3$ with $C_{\infty}$-coefficients having compact support in $\Omega$, with respect to the norm $\|\cdot\|$ induced by the inner product

$$
(u, v)=\sum_{q=0}^{3}\left(u_{q}, v_{q}\right)_{q},
$$

where $u=u_{0}+u_{1}+u_{2}+u_{3}, v=v_{0}+v_{1}+v_{2}+v_{3}, u_{q}, v_{q}$ denoting $q$-forms with compactly supported and smooth coefficients. We will denote the set of such differential forms by $\stackrel{\circ}{C}_{\infty}(\Omega)$.

The precise meaning of equations (0.9)-(0.11) will be established in a suitable functional-analytic setting based on $L_{2}(\Omega)$ and under suitable assumptions, in particular for the nonlinearity $\varepsilon$. It is clear that the framework described here can be generalized further. The necessary ideas may be found in [11]. Here, however, we shall focus on the application problem at hand. In a certain sense the present paper is a continuation of the work in [6], the difference being that we here will be dealing with more general material relations allowing for a coupling of electric and magnetic effects.

In the first section we shall develop the main tools for the solution theory that is given in Section 2. Section 1 will also provide the necessary terminology to formulate $(0.9)-(0.11)$ in a more precise way. The main results are contained in the main theorem and the associated corollary (existence, uniqueness and continuous dependence of solution). General references for notations and for more details on differential forms are e.g. [4], [12]. 
1. Basic concepts and formulation of problem. To introduce suitable Hilbert spaces to interpret the boundary value problem of electro- and magnetostatics we shall employ for convenience a definition scheme introduced in [10].

Let $d, \delta$ denote the formal exterior derivative and its co-derivative respectively. They are both densely defined and closable as operators, say $\stackrel{\circ}{d}, \stackrel{\circ}{\delta}$, defined on $\check{C}_{\infty}(\Omega)$. Thus, we may extend the meaning of differentiability to be generalized in the weak sense, which in the present context means

$$
\delta=-\stackrel{\circ}{d}^{*} \quad \text { on } D\left(\stackrel{\AA}{d}^{*}\right), \quad d=-\stackrel{\circ}{\delta}^{*} \quad \text { on } D\left(\AA^{*}\right) .
$$

Since $d, \delta$ thus extend $\stackrel{\circ}{d}, \delta$ we may use $d, \delta$ as the standard notation. Which operator is intended can be seen from a look at the domain. In this framework we define the Hilbert spaces

$$
\begin{aligned}
H(d, \Omega) & :=\left\{\psi \in L_{2}(\Omega) \mid d \psi \in L_{2}(\Omega)\right\} \equiv D\left(\stackrel{\circ}{\delta}^{*}\right), \\
H(\delta, \Omega) & :=\left\{\psi \in L_{2}(\Omega) \mid \delta \psi \in L_{2}(\Omega)\right\} \equiv D\left(\stackrel{\circ}{d}^{*}\right), \\
\stackrel{\circ}{H}(d, \Omega) & :=\{\psi \in H(d, \Omega) \mid(d \psi, \chi)+(\psi, \delta \chi)=0 \text { for all } \chi \in H(\delta, \Omega)\}, \\
\stackrel{\circ}{H}(\delta, \Omega) & :=\{\psi \in H(\delta, \Omega) \mid(\delta \psi, \chi)+(\psi, d \chi)=0 \text { for all } \chi \in H(d, \Omega)\},
\end{aligned}
$$

where the respective graph inner products

$$
(\cdot, \cdot)+(d \cdot, d \cdot), \quad(\cdot, \cdot)+(\delta \cdot, \delta \cdot)
$$

are used as the underlying inner products. Apparently, we have

$$
\stackrel{\circ}{H}(d, \Omega) \subset H(d, \Omega),
$$

i.e. $\stackrel{\circ}{H}(d, \Omega)$ is a subspace of $H(d, \Omega)$. By Hodge star duality we also have

$$
\stackrel{\circ}{H}(\delta, \Omega) \subset H(\delta, \Omega) .
$$

Note that the Hodge star operator is a unitary automorphism of $L_{2}(\Omega)$.

Now let $\Phi$ stand for either $d$ or $\delta$, and if $\Phi$ denotes one, then $\Phi^{\prime}$ denotes the other. With this notation we also introduce the subspaces

$$
\begin{aligned}
H_{0}(\Phi, \Omega) & =\{\psi \in H(\Phi, \Omega) \mid \Phi \psi=0\}, \\
\stackrel{\circ}{H}_{0}(\Phi, \Omega) & =\{\psi \in \stackrel{\circ}{H}(\Phi, \Omega) \mid \Phi \psi=0\},
\end{aligned}
$$

and

$$
\stackrel{\circ}{H}_{0}\left(\Phi, \Phi^{\prime}, \Omega\right)=\left\{\psi \in \stackrel{\circ}{H}_{0}(\Phi, \Omega) \mid \Phi^{\prime} \psi=0\right\}=\stackrel{\circ}{H}_{0}(\Phi, \Omega) \cap H_{0}\left(\Phi^{\prime}, \Omega\right) .
$$

The latter spaces (i.e. those defined in (1.7)) are known as the spaces of harmonic differential forms. By Hodge star duality they are the same for both choices. With the same convention we introduce another two Hilbert spaces:

$$
\stackrel{\circ}{H}\left(\Phi, \Phi^{\prime}, \Omega\right)=\stackrel{\circ}{H}(\Phi, \Omega) \cap H\left(\Phi^{\prime}, \Omega\right),
$$

with the inner product

$$
(\cdot, \cdot)+(\Phi \cdot, \Phi \cdot)+\left(\Phi^{\prime} \cdot, \Phi^{\prime} \cdot\right) .
$$


These are unitarily equivalent. The Hodge star operator induces a unitary mapping between $\stackrel{\circ}{H}(d, \delta)$ and $\stackrel{\circ}{H}(\delta, d)$.

We shall omit the reference to the domain $\Omega$ since it will be clear from the context.

Within the language developed so far we may describe the set where we shall seek solutions $\omega$ of $(0.9)-(0.11)$, in the following way:

$$
\omega \in \stackrel{\circ}{H}(d), \quad \varepsilon(\omega) \in H(\delta) .
$$

Here $\varepsilon$ is inherited from (0.11) as far as the 1-form and 2-form parts of $\omega$ (and $\eta$ ) are concerned. We assume that (0.11) is trivially extended to 0 -forms and 3 -forms as the identity and continue to use the same notation for this extended "material" relation. We note that the condition $\omega \in \stackrel{\circ}{H}(d)$ generalizes the boundary condition (0.10). In the following we may use the classical notation " $\omega=0$ on $\partial \Omega$ " in this generalized interpretation. It should be kept in mind, however, that this generalization of the boundary condition needs no restriction on the regularity of the boundary.

A simple application of the projection theorem for Hilbert spaces leads to our first decomposition lemma (for a proof, compare [8]).

LEMMA 1. We have the following orthogonal decompositions of $L_{2}(\Omega)$ :

$$
\begin{aligned}
L_{2}(\Omega) & =\overline{d \stackrel{\circ}{H}(d)} \oplus \stackrel{\circ}{H}_{0}(d, \delta) \oplus \overline{\delta H(\delta)} \\
& =\overline{d \stackrel{\circ}{H}(d)} \oplus H_{0}(\delta) \\
& =\stackrel{\circ}{H}_{0}(d) \oplus \overline{\delta H(\delta)}
\end{aligned}
$$

and

$$
\begin{aligned}
L_{2}(\Omega) & =\overline{d H(d)} \oplus \stackrel{\circ}{H}_{0}(\delta, d) \oplus \overline{\delta \stackrel{\circ}{H}(\delta)} \\
& =\overline{d H(d)} \oplus \stackrel{\circ}{H}_{0}(\delta) \\
& =H_{0}(d) \oplus \overline{\delta \stackrel{\circ}{H}(\delta)} .
\end{aligned}
$$

Note that (1.12) follows from (1.11) by Hodge star duality.

As an immediate consequence of Lemma 1 and the definition of the graph spaces of $d, \delta$, we have the following decomposition result.

LEMma 2. The Hilbert spaces $H(d), \stackrel{\circ}{H}(d)$ allow the following representations as direct sums:

$$
\begin{aligned}
H(d) & =\overline{d H(d)} \oplus \overline{\stackrel{\circ}{H}_{0}(\delta, d) \oplus \overline{\delta \stackrel{\circ}{H}(\delta)} \cap H(d)} \\
& =H_{0}(d) \oplus \overline{\delta \stackrel{\circ}{H}(\delta)} \cap H(d) \\
& =\overline{d H(d)} \oplus \stackrel{\circ}{H}_{0}(\delta) \cap H(d)
\end{aligned}
$$

and

$$
\stackrel{\circ}{H}(d)=\overline{d \stackrel{\circ}{H}(d)} \oplus \stackrel{\circ}{H}_{0}(d, \delta) \oplus \overline{\delta H(\delta)} \cap \stackrel{\circ}{H}(d)
$$




$$
\begin{aligned}
& =\stackrel{\circ}{H}_{0}(d) \oplus \overline{\delta H(\delta)} \cap \stackrel{\circ}{H}(d) \\
& =\overline{d \stackrel{\circ}{H}(d)} \oplus H_{0}(\delta) \cap \stackrel{\circ}{H}(d) .
\end{aligned}
$$

Moreover, the dual results for $H(\delta)$ and $\stackrel{\circ}{H}(\delta)$ are valid:

$$
\begin{aligned}
H(\delta) & =\overline{\delta H(\delta)} \oplus \stackrel{\circ}{H}_{0}(d, \delta) \oplus \overline{d \stackrel{\circ}{H}(d)} \cap H(\delta) \\
& =H_{0}(\delta) \oplus \overline{d \stackrel{\circ}{H}(d)} \cap H(\delta) \\
& =\overline{\delta H(\delta)} \oplus \stackrel{\circ}{H}_{0}(d) \cap H(\delta)
\end{aligned}
$$

and

$$
\begin{aligned}
\stackrel{\circ}{H}(\delta) & =\overline{\delta \stackrel{\circ}{H}(\delta)} \oplus \stackrel{\circ}{H}_{0}(\delta, d) \oplus \overline{d H(d)} \cap \stackrel{\circ}{H}(\delta) \\
& =\stackrel{\circ}{H}_{0}(\delta) \oplus \overline{d H(d)} \cap \stackrel{\circ}{H}(\delta) \\
& =\overline{\delta \circ}(\delta) \oplus H_{0}(d) \cap \stackrel{\circ}{H}(\delta) .
\end{aligned}
$$

The decompositions are orthogonal with respect to the inner product of $H(d)$ and $H(\delta)$, respectively.

Note that the dual result can be obtained by exchanging the role of $d$ and $\delta$. This allows us to be brief about the dual statement and we shall do so henceforth. Lemma 2 implies the following result.

LEMMA 3. We have

$$
d H(d)=d(\overline{\delta \stackrel{\circ}{H}(\delta)} \cap H(d)), \quad d \stackrel{\circ}{H}(d)=d(\overline{\delta H(\delta)} \cap \stackrel{\circ}{H}(d)) .
$$

The dual results are also valid.

Another consequence of Lemma 2 is the following density result.

Lemma 4. The set $H(d) \cap \overline{\delta \stackrel{\circ}{H}(\delta)}$ is dense in the subspace $\overline{\delta \stackrel{\circ}{H}(\delta)}$ of $L_{2}(\Omega)$. Similarly, $\stackrel{\circ}{H}(d) \cap \overline{\delta H(\delta)}$ is dense in the subspace $\overline{\delta H(\delta)}$ of $L_{2}(\Omega)$.

Proof. Noting that $\stackrel{\circ}{H}(d)$ (and therefore $H(d)$ ) is dense in $L_{2}(\Omega)$, we only have to apply the decompositions of Lemma 2 . The respective projections are continuous (in the space $L_{2}(\Omega)$ ) and therefore the desired result follows.

To proceed we have to be more specific about assumptions on the boundary of $\Omega$. We shall assume that

(1.17) $\quad M$ is an open, bounded domain of $\mathbb{R}^{3}$ which is also a Lipschitz manifold with boundary.

According to [9] our assumption on $\Omega$ guarantees the following compactness results:

$$
\stackrel{\circ}{H}(d, \delta) \hookrightarrow \hookrightarrow L_{2}(\Omega), \quad \stackrel{\circ}{H}(\delta, d) \hookrightarrow \hookrightarrow L_{2}(\Omega) .
$$

An immediate consequence of these imbedding results is that the unit sphere in $\stackrel{\circ}{H}_{0}(d, \delta)$ is compact and therefore that $\stackrel{\circ}{H}_{0}(d, \delta)$ is finite-dimensional. Let $\stackrel{\mathcal{B}}{\text { be a }}$ 
fixed (finite) basis of the harmonic space $\stackrel{\circ}{H}_{0}(d, \delta)$. Without loss of generality we may assume that the elements of $\stackrel{\circ}{\mathcal{B}}$ are differential forms of specific degrees (rather than general linear combinations of these). As another consequence of (1.18) we have

LEMMA 5. There is a uniform constant $C$ such that

$$
\|\omega\| \leq C\left(\|d \omega\|+\|\delta \omega\|+\sum_{\psi \in \mathcal{B}}|(\omega, \psi)|\right),
$$

for all $\omega \in \stackrel{\circ}{H}(d, \delta)$ or $\omega \in \stackrel{\circ}{H}(\delta, d)$.

Proof. The estimate can be shown by a standard contradiction argument making use of the compact imbedding results (1.18) (see [2], [8]).

Lemma 5 implies the following closedness results.

LEMMA 6. The following spaces are closed subspaces of $L_{2}(\Omega)$ :

$$
d H(d)=d(\delta \stackrel{\circ}{H}(\delta) \cap H(d)), \quad d \stackrel{\circ}{H}(d)=d(\delta H(\delta) \cap \stackrel{\circ}{H}(d)),
$$

and by the Hodge * duality

$$
\delta H(\delta)=\delta(d \stackrel{\circ}{H}(d) \cap H(\delta)), \quad \delta \stackrel{\circ}{H}(\delta)=\delta(d H(d) \cap \stackrel{\circ}{H}(\delta)) .
$$

Proof. It suffices to consider the first case. The second closedness result follows analogously. The rest of the lemma is obtained by star duality.

Applying the previous lemma to sequences $\left(\phi_{n}\right)_{n}$ in $\overline{\delta \stackrel{\circ}{H}(\delta)} \cap H(d)$, or $\overline{\delta H(\delta)} \cap \stackrel{\circ}{H}(d)$ respectively, yields the convergence of $\left(\phi_{n}\right)_{n}$ in $L_{2}(\Omega)$. Note

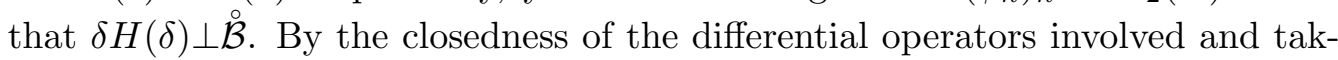
ing Lemma 3 into account this shows the closedness of the spaces in (1.20) and also that the closure bars can be omitted in Lemma 3.

The above results suggest the following reformulation of our initial problem.

Boundary value problem of electro-magnetostatics: Find $\omega \in \stackrel{\circ}{H}(d)$ such that $\varepsilon(\omega) \in H(\delta)$ and

$$
d \omega=f, \quad \delta \varepsilon(\omega)=g,
$$

where $f, g \in L_{2}(\Omega)$ are given.

As necessary solvability conditions we obtain immediately from Lemma 1:

$$
\begin{gathered}
f \in \stackrel{\circ}{H}_{0}(d), \quad g \in H_{0}(\delta), \\
f, g \perp \stackrel{\circ}{H}_{0}(d, \delta) .
\end{gathered}
$$

Note that a 0 -form in $\stackrel{\circ}{H}_{0}(d)$ is necessarily the zero function, and a 3-form in $H_{0}(\delta)$ is necessarily a constant multiple of the volume form, which in conjunction with (1.24) implies that this 3-form must also be zero. Therefore, $f$ does not contain any 0 -form component and $g$ does not contain any 3 -form part. 
The solution theory of (1.22) will be obtained by a now straightforward application of the theory of monotone operators (see e.g. [1], [5]). This will be the focus of the next section. We continue to follow the ideas described in [11] and will therefore permit ourselves to be brief.

2. Solution theory of non-linear EM-statics. In order to solve problem (1.22) we shall reformulate it as an abstract equation of the form

$$
A(u)=F,
$$

where $u$ is to be found in a certain Hilbert space $V$ continuously and densely imbedded into another Hilbert space $H$ (with norm $\|\cdot\|$ and inner product $(\cdot, \cdot)$ ), $F$ is a given right-hand side in the normed dual $V^{\prime}$ of $V$ and $A$ is a monotone operator. Thus, by the usual identification of $H$ with its dual, we have a Gelfand triple

$$
V \hookrightarrow H \hookrightarrow V^{\prime}
$$

and $A$ maps $V$ into $V^{\prime}$.

The operator $A$ is usually given by a continuous form

$$
\alpha: V \times V \rightarrow \mathbb{R},
$$

via the relations

$$
\langle A(u), v\rangle=\alpha(u, v) \quad \text { for all } v \in V,
$$

and

$u \in D(A):=\{u \in V \mid \alpha(u, \cdot)$ is a continuous linear functional on $V\}$.

Consequently, (2.1) may be written in variational form

$$
\alpha(u, v)=\langle F, v\rangle \quad \text { for all } v \in V .
$$

We recall from [5] that solvability of problems $(2.1),(2.4)$ is assured by the following

Proposition. Let $A$ (or $\alpha$ ) be a strongly monotone and coercive operator (or form), i.e. there is a constant $\vartheta>0$ such that

$$
\begin{gathered}
\langle A(u)-A(v), u-v\rangle \geq \vartheta\|u-v\|^{2} \\
\left(\text { or } \alpha(u, u-v)-\alpha(v, u-v) \geq \vartheta\|u-v\|^{2}\right), \text { and } \\
\langle A(u), u\rangle \geq \vartheta\|u\|^{2}
\end{gathered}
$$

(or $\left.\alpha(u, u) \geq \vartheta\|u\|^{2}\right)$, for all $u, v \in V$. Then there exists a unique solution of $(2.1)$ (or $(2.4))$.

In order to satisfy the assumption of this proposition we constrain ourselves to a certain class of non-linearities.

Assumption. $\varepsilon$ is a bijection such that $\varepsilon^{-1}$ is a Lipschitz continuous and strongly monotone mapping from $L_{2}(\Omega)$ to $L_{2}(\Omega)$ (with $\varepsilon(0)=0$ for simplicity). 
In order to obtain uniqueness of the solution of (1.22) we have to impose a finite number of additional conditions:

$$
(\psi, \varepsilon(\omega))=c_{\psi} \quad \text { for all } \psi \in \stackrel{\circ}{\mathcal{B}},
$$

where $c_{\psi}, \psi \in \stackrel{\circ}{\mathcal{B}}$, are given real numbers.

We shall see that conditions $(1.23),(1.24)$ are indeed sufficient to obtain a solution of problem (1.22). Unique existence follows for solutions satisfying conditions of the type (2.5).

In order to find a solution we decompose $\omega$ according to Lemma 1 and taking Lemma 6 into account:

$$
\varepsilon(\omega)=d \varphi_{0}+h_{0}+\delta \Phi,
$$

where

$$
\begin{gathered}
\varphi_{0} \in \stackrel{\circ}{H}(d) \cap \delta H(\delta), \quad h_{0} \in \stackrel{\circ}{H}_{0}(d, \delta), \\
\Phi \in H(\delta) \cap d \stackrel{\circ}{H}(d) .
\end{gathered}
$$

Since $h+\delta \phi$ is in $H_{0}(\delta), \varphi_{0} \in \stackrel{\circ}{H}(d) \cap \delta H(\delta)$ is determined as the unique solution of the boundary value problem

$$
\delta d \varphi_{0}=\delta \varepsilon(\omega)=g,
$$

and is thus determined by the given $g$ alone. Uniqueness and existence follows by applying Riesz' representation theorem to the space $\stackrel{\circ}{H}(d) \cap \delta H(d)$ (with norm $\|d \cdot\|)$.

Moreover, since

$$
\delta H(\delta) \perp \stackrel{\circ}{\mathcal{B}}
$$

we have by $(2.5)$

$$
\left(\psi, h_{0}\right)=c_{\psi}-\left(\psi, d \varphi_{0}\right) \quad \text { for all } \psi \in \stackrel{\circ}{\mathcal{B}}
$$

We can see that (2.11) determines $h_{0}$ uniquely in terms of the given $c_{\psi}, \psi \in \dot{\mathcal{B}}$, and the previously determined $\varphi_{0}$ (choosing $\stackrel{\circ}{\mathcal{B}}$ as an orthonormal basis of $\stackrel{\circ}{H}_{0}(d, \delta)$ makes this obvious). Thus it remains to find $\phi$.

R e m ark. The idea used here is of course just a variant of the usual approach to solve the problem by introducing potentials. The role of potential is played by $\phi$. The basic idea of this reduction is quite natural and has been applied previously (for the electrostatic problem) in the particular way employed here by D. Graffi (see [3], pp. 11-14).

From (1.22) we have

$$
d \varepsilon^{-1}\left(\delta \phi+\left(h_{0}+d \varphi_{0}\right)\right)=f .
$$

Choose

$$
H:=d \stackrel{\circ}{H}(d), \quad V=H(\delta) \cap d \stackrel{\circ}{H}(d),
$$


and define

$$
\alpha(u, v)=\left(\varepsilon^{-1}\left(\delta u+\beta_{0}\right)-\varepsilon^{-1}\left(\beta_{0}\right), \delta v\right)
$$

for $u, v \in V$, where

$$
\beta_{0}=h_{0}+d \varphi_{0} .
$$

The density of $V$ in $H$ follows according to Lemma 4. Due to the assumption on $\varepsilon$ we see that $\alpha$ is strongly monotone and coercive, so that the Proposition can be applied; i.e. we get a unique solution $\phi \in V$ of

$$
\alpha(\phi, v)=(f, v)-\left(\varepsilon^{-1}\left(\beta_{0}\right), \delta v\right) \quad \text { for all } v \in V .
$$

Note that the right-hand side of (2.15) is indeed a continuous, linear functional in $V^{\prime}$. The solution $\phi$ of (2.15) apparently also satisfies

$$
\left(\varepsilon^{-1}\left(\delta \phi+\beta_{0}\right), \delta v\right)=(f, v),
$$

which in turn implies

$$
\omega:=\varepsilon^{-1}\left(\delta \phi+\beta_{0}\right) \in \stackrel{\circ}{H}(d) .
$$

This $\omega$ apparently solves problem (1.22), (2.5), proving existence. Uniqueness of the solution of (1.22), (2.5) is clear since in fact - as demonstrated - this problem is equivalent to $(2.9),(2.11),(2.12)$ and the latter is equivalent to its variational formulation (2.15). Thus, we obtain our main theorem.

THEOREM. The boundary value problem (1.22), (2.5) has a unique solution $\omega$ iff conditions (1.23), (1.24) are satisfied.

It should be clear that if $f=j, g=q$ and $c_{\psi}=0$ for 0 -forms and 3-forms $\psi$ in $\mathcal{B}$ it follows that $\omega$ and $\eta$ have no (non-trivial) 0 -form and 3 -form parts (compare the arguments used for $f, g$ above). In this case the problem reduces to our original problem.

The existence part of the standard proof of the main theorem involves the a priori estimate

$$
\begin{aligned}
\vartheta\|\delta \phi\|^{2} & \leq\left(\varepsilon^{-1}\left(\delta \phi+\beta_{0}\right)-\varepsilon^{-1}\left(\beta_{0}\right),\left(\delta \phi+\beta_{0}\right)-\beta_{0}\right) \\
& \equiv \alpha(\phi, \phi)=(f, \phi)-\left(\varepsilon^{-1}\left(\beta_{0}\right), \delta \phi\right) \\
& \leq\|f\|\|\phi\|+\left\|\varepsilon^{-1}\left(\beta_{0}\right)\right\|\|\delta \phi\| \leq\left(C\|f\|+\left\|\varepsilon^{-1}\left(\beta_{0}\right)\right\|\right)\|\delta \phi\|
\end{aligned}
$$

for the solution $\phi$ of (2.15). Here we have used Lemma 5 in the last step. Thus we obtain

$$
\|\delta \phi\| \leq C\left(\|f\|+\left\|\varepsilon^{-1}\left(\beta_{0}\right)\right\|\right)
$$

for the solution $\phi$. By the properties of $\varepsilon^{-1}$ and the definition of $\beta_{0}$ we obtain the a priori estimate

$$
\|\phi\| \leq C\left(\|f\|+\|g\|+\left[\sum_{\psi \in \mathcal{B}}\left|c_{\psi}\right|^{2}\right]^{1 / 2}\right),
$$


where the constant $C>0$ does not depend on the data. The estimate $(2.16)$ can be used to derive an estimate for the original solution $\omega$ :

$$
\|\omega\| \leq C_{0}\left(\|f\|+\|g\|+\left[\sum_{\psi \in \mathcal{B}}\left|c_{\psi}\right|^{2}\right]^{1 / 2}\right),
$$

where the generic constant $C_{0}>0$ does not depend on the data $f, g$, and $c_{\psi}$, $\psi \in \stackrel{\circ}{\mathcal{B}}$. This yields the following corollary to the main theorem.

Corollary. The solution $\omega$ of (1.22), (2.5) depends continuously on the data in the sense of (2.18). A corresponding result holds for the solution $\phi$ of (2.15).

For further generalizations and extensions of these results we refer the interested reader to [6] and [11].

\section{References}

[1] H. Brezis, Opérateurs Maximaux Monotones, North-Holland, Amsterdam 1973.

[2] K. O. Friedrichs, Differential forms on Riemannian manifolds, Comm. Pure Appl. Math. 8 (1955), 551-590.

[3] D. Graffi, Nonlinear Partial Differential Equations in Physical Problems, Pitman, Boston 1980.

[4] N. J. Hicks, Notes on Differential Geometry, Van Nostrand, Princeton 1965.

[5] J. L. Lions, Quelques méthodes de résolution des problèmes aux limites non linéaires, Dunod-Gauthier-Villars, Paris 1969.

[6] A. Milani and R. Picard, Decomposition theorems and their application to non-linear electro- and magneto-static boundary value problems, in: Partial Differential Equations and Calculus of Variations, Lecture Notes in Math. 1357, Springer, Berlin 1988, 317-340.

[7] R. Picard, Randwertaufgaben der verallgemeinerten Potentialtheorie, Math. Methods Appl. Sci. 3 (1981), 218-228.

[8] -, On the boundary value problems of electro- and magnetostatics, Proc. Roy. Soc. Edinburgh 92A (1982), 165-174.

[9] —, An elementary proof for a compact imbedding result in generalized electromagnetic theory, Math. Z. 187 (1984), 151-164.

[10] - The low frequency limit for time-harmonic acoustic waves, Math. Methods Appl. Sci. 8 (1986), 436-450.

[11] - Some decomposition theorems and their application to non-linear potential theory and Hodge theory, ibid. 12 (1990), 35-52.

[12] C. von Westenholz, Differential Forms in Mathematical Physics, Stud. in Math. Appl., North-Holland, Amsterdam 1978 